\title{
Behandlung von Muskelverletzungen
}

\author{
Frieder Mauch
}

\section{Zusammenfassung}

Muskelverletzungen stellen die häufigste Sportverletzung dar. Die 4 großen Muskelgruppen (Hamstrings, Adduktoren, Gastroknemius und die Kniestrecker) stehen hier im Mittelpunkt der Behandlung. Meist handelt es sich um akute Verletzungen, die durch exzentrische Krafteinwirkungen entstehen. Die Diagnose beginnt mit einer genauen Anamneseerhebung des Traumas, gefolgt von einer exakten klinischen Untersuchung. Die Bildgebung mit Sonografie und Kernspintomografie (MRT) stellt einen wichtigen Baustein in der Diagnostik dar mit dem Ziel, die strukturelle Verletzung von der funktionellen Störung zu unterscheiden und das Ausmaß der Verletzung festzustellen. Die häufigste Therapieoption in der Behandlung der Muskelverletzung stellt die konservative Therapie dar und orientiert sich an den Phasen der Muskelheilung. In vielen Fällen (Freizeitsport) reicht eine konservative Therapie nach dem RICE-Prinzip mit nachfolgender beschwerdeabhängiger Aufbelastung aus um die Rückkehr zum Sport zu gewährleisten. Die Infiltrationstherapie einschließlich Platetrich-Plasma (PRP) kann eine Therapieoption darstellen, soll aber nicht zur Beschleunigung des Heilungsprozesses eingesetzt werden. Die operative Versorgung von Muskelverletzungen ist seltenen Indikationen vorbehalten.

\section{Treatment of Muscle Lesions}

The commonest sporting injuries are muscle injuries. Hamstrings, adductors, gastrocnemius and the knee extensor muscles are most often affected. Most injuries occur during eccentric tension impact. Diagnostic testing begins with taking the exact medical history and a detailed clinical investigation. Imaging with ultrasound and MRI is important for differentiation between structural lesions and functional disorders and determines the extent of the injury. Treatment is most often conservative and depends on the three phases of the healing process. In most cases (leisure sports) the RICE concept with subsequent pain-adapted load increase suffices for a return to sport. Infiltration therapy, including platelet-rich plasma (PRP), is an additional therapeutic option, but should not be used to accelerate the healing process. It is only rarely that surgery is needed to treat muscle injuries.
Muskelverletzungen stellen die häufigste Sportverletzung dar. Abhängig von der Lokalisation und Schwere der Verletzung stehen unterschiedliche Therapieoptionen zur Verfügung.

OP-JOURNAL 2016; 32: 27-33

(c) Georg Thieme Verlag KG Stuttgart · New York DOI http://dx.doi.org/10.1055/s-0035-1568629
Die 4 großen Muskelgruppen (Hamstrings, Adduktoren, Gastroknemius und die Kniestrecker) stehen im Mittelpunkt der Behandlung [11]. Meist sind es akute Verletzungen, die durch exzentrische Krafteinwirkungen entstehen, die 1,911,5-mal höher sein können als die konzentrische Krafteinwirkung [19]. Hierdurch entstehen indirekte Muskelverletzungen unterschiedlichen Ausmaßes, vor allem am Muskel-Sehnen-Übergang. Die meisten Muskelverletzungen sind indirekte Partialrupturen (Minorrupturen) und heilen ohne Narbenbildung aus. Die höhergradige Muskelverletzung heilt im Sinne eines reparativen Prozesses mit Ausbildung einer mehr oder weniger stark ausgebildeten Narbe [19]. Hier werden 3 Phasen unterschieden, die sich auch in den einzelnen Abschnitten der konservativen Therapiekonzepte wiederfinden (Abb. 1).

- Verletzungsphase/Entzündungsphase (1.-5. Tag): Sie ist durch die Ruptur der Muskelzellen mit darauffolgender Ausbildung eines Hämatoms und der Einleitung einer entzündlichen Reaktion charakterisiert.

- Reparationsphase (ca. 5.-14. Tag): Hier steht die Phagozytose mit der Regeneration der Muskelzellen und der beginnenden Ausbildung eines Narbengewebes im Mittelpunkt.

- Remodeling-Phase (14.-21. Tag und länger): Ausbildung und Reorganisation der Narbe mit „kompletter“ Regeneration der ehemaligen Muskelverletzung und Erreichen der funktionellen Leistungsfähigkeit.

\section{Klinik/Bildgebung und Klassifikation}

Die Diagnose der Muskelverletzung beginnt mit einer genauen Anamneseerhebung des Traumas, gefolgt von einer exakten klinischen Untersuchung, einschließlich Inspektion und Palpation. Die Funktionsdiagnostik steht am Ende [19].

Unterschiedliche Klassifikationen sind in der Literatur veröffentlicht. Klinische Klassifikationen mit 3 Graden sind weit verbreitet. Milde Formen stellen hier Grad-I-Verletzungen mit nur geringem strukturellem Schaden, geringer Schwellung und wenig Schmerzen, begleitet von geringem Kraftverlust und Bewegungseinschränkung dar. Grad II sind moderate Verletzungen mit größerem strukturellem Schaden und deutlichem 
2

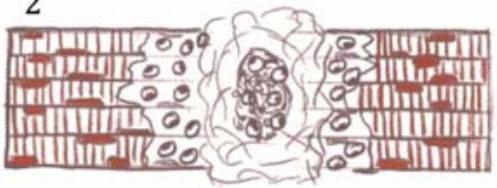

5

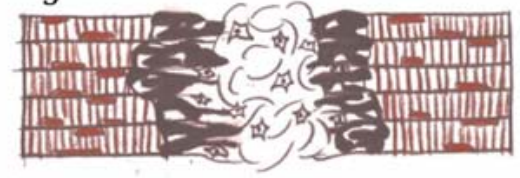

\section{4}

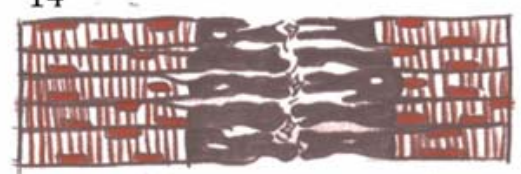

3

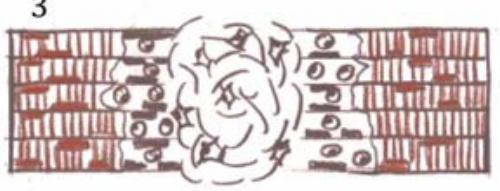

7

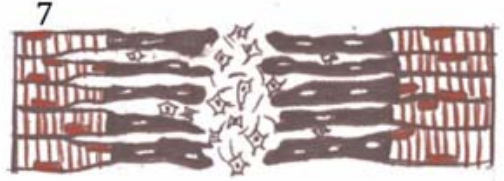

21

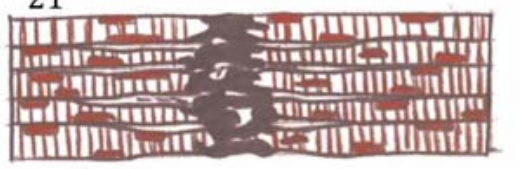

Abb. 1 Schematische Darstellung der Muskelheilung.

Tab. 1 Aktuelle Klassifikation der Muskelverletzungen nach Mueller-Wohlfahrt et al.: Konsensmeeting von 15 internationalen Experten.

\begin{tabular}{|c|c|c|}
\hline \multirow{11}{*}{$\begin{array}{l}\text { A. Indirect } \\
\text { Muscle Disor- } \\
\text { ders/Injury }\end{array}$} & \multirow{6}{*}{$\begin{array}{l}\text { Functional } \\
\text { Muscle } \\
\text { Disorders }\end{array}$} & Type 1: Overexertion-related Muscle Disorders \\
\hline & & Type 1A: Fatique-induced Muscle Disorders \\
\hline & & Type 1B: Delayed-onset Muscle Sorenesse (DOMS) \\
\hline & & Type 2: Neuromuscular Muscle Disorders \\
\hline & & Type 2A: Spine-related Muscle Disorders \\
\hline & & Type 2B: Muscle-related Muscle Disorders \\
\hline & \multirow{5}{*}{$\begin{array}{l}\text { Structural } \\
\text { Muscle Injury }\end{array}$} & Type 3: Partial Muscle Tear \\
\hline & & Type 3A: Minor Partial Tear \\
\hline & & Type 3B: Moderate Partial Tear \\
\hline & & Type 4: (Sub)total Tear \\
\hline & & Subtotal or complete Muscle Tear and Tendinous Avulsion \\
\hline \multirow{2}{*}{$\begin{array}{l}\text { B. Direct Mus- } \\
\text { cle Disorders }\end{array}$} & & Contusion \\
\hline & & Laceration \\
\hline
\end{tabular}

Funktionsverlust, während eine komplette Ruptur des Muskels einen drittgradigen Schaden darstellt [19]. Subtypen der Muskelverletzungen und eine genaue Terminologie werden hier nicht erfasst. Mueller-Wohlfahrt et al. schlagen deshalb ein neues Klassifikationssystem vor [30]. Unterschieden werden hier akute strukturelle Schäden von der akuten funktionellen Störung. Symptome, klinische Untersuchung, Lokalisation und Bildgebung sind für die Unterscheidung der Gruppen notwendig. Die meisten indirekten Muskelverletzungen stellen Partialrupturen dar, die von dieser Arbeitsgruppe noch in Minor-/moderate Verletzungen unterteilt werden (Tab. 1).

\section{Bildgebung}

Die Bildgebung mit Sonografie und Kernspintomografie (MRT) stellt einen wichtigen Baustein in der Diagnostik dar. Der frühe Einsatz der Sonografie kann schnell und ohne großen Aufwand den möglichen strukturellen Schaden erfassen [35]. Die MRT-Diagnostik bietet den Vorteil der exakten Darstellung mit Lokalisation und Ausmaß der Verletzung mit Darstellung eines Ödems bzw. des strukturellen Schadens selbst. In Kombination mit der Klinik lässt sich die Verletzung meist schnell und sicher erfassen [10]. Ein weiterer Vorteil der Kernspintomografie ist die sichere Erfassung von Differenzialdiagnosen im Hüft- bereich wie Stressfrakturen (Abb. 2), Labrumverletzungen, Avulsionsverletzungen, „Greater Trochanter Pain Syndrome“.

\section{Therapiekonzepte}

Die vorherrschenden Therapieprinzipien der Muskelverletzungen sind noch nicht vollständig geprüft und es fehlen z.T. wissenschaftliche Studien zur genauen Evaluation $[19,29]$. In dem folgenden Abschnitt sollen „klassische“ Therapieprinzipien auf der Basis von prinzipiellen Überlegungen zur Muskelheilung bei strukturellen Verletzungsformen besprochen werden. Da die HamstringGruppe am häufigsten verletzt ist, wird sie hier etwas genauer betrachtet.

\section{Konservative Therapiemaßnahmen}

Die häufigste Therapieoption in der Behandlung der Muskelverletzung stellt die konservative Therapie dar und sollte sich, wie oben schon erwähnt, an den 3 Phasen der Muskelheilung orientieren $[19,42]$. Der Sportler muss, unabhängig von der Schwere der Verletzung, genügend „Zeit“ bekommen, die Akutphase, Subakutphase/Regenerationsphase und funktionelle Phase zu durchlaufen, um eine ausreichende Regeneration der Verletzung zu erreichen. Das Ziel der einzelnen Trainingsphasen liegt in der Entwicklung der notwenigen funktionellen Fähigkeiten für den Sportler, um die erneute Verletzung zu verhindern. Die Ausfallzeiten orientieren sich an der Schwere und Klassifikation der Muskelverletzung (siehe Tab. 1).

\section{Akutphase}

\section{Immobilisation/Mobilisation}

In den ersten Tagen ist eine kurzzeitige „Ruhigstellung“ der Extremität mithilfe einer elastokompressiven Wickelung gefolgt von einer Frühmobilisation sinnvoll (Abb.3). Je nach Ausmaß der Verletzung müssen zusätzlich Gehhilfen benutzt werden [18]. Diese Zeit benötigt die erste Ausbildung der Narbe zur Überbrückung der „Lücke“ zwischen den Stümpfen, um der kontraktilen Kraft dann entgegenwirken zu können [18]. Vorsichtige Massage um die verletzte Stelle des Muskels und der Lendenwirbelsäule sind hilfreich [33]. Ebenso können Infiltrationen von Lokalanästhetikum, Actovegin, Traumeel S und Platelet-rich Plasma (PRP) adjuvant in dieser Phase angewandt werden. Die Rolle der NSAR ist noch 


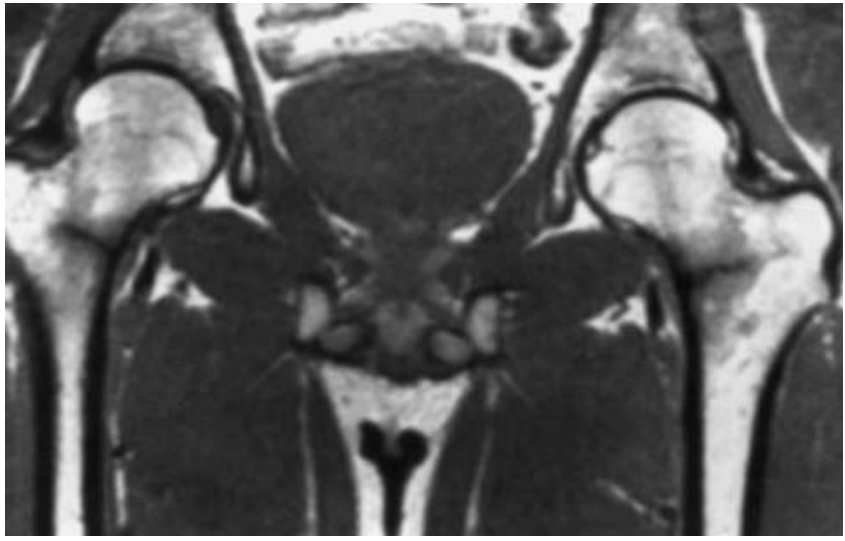

nicht abschließend geklärt. Insgesamt kann die Gabe kurzzeitig in der Akutphase sinnvoll sein. Der systematische Einsatz wird aber unter Experten in den letzten Jahren abgelehnt [34].

\section{RICE-Prinzip}

Sinnvoll sofort nach Verletzung einzuleiten und bis zu 24 Stunden nach Verletzung weiterzuführen: Alle 4 RICE-Maßnahmen (Rest/Ice/Compression/Evaluation) haben zum Ziel, die Retraktion der Muskelstümpfe und die Einblutung an der verletzten Stelle möglichst gering zu
Abb. 2 Koronare SE-T1-Aufnahmen von einem Marathonläufer mit beidseitiger Stressfraktur. halten, um somit eine Ausbildung einer kleinen Narbe zu erreichen. Die Eisanwendung erfolgt mit einer Dauer von 15-20 Minuten mit Intervallen von 3060 Minuten, gefolgt von einer elastokompressiven Wickelung mit Kurzzugbinden [2]. Die Gabe von NSAR kann in der Akutphase sinnvoll sein. Zusätzlich können Tauchbäder für 10 Minuten bei $25^{\circ} \mathrm{C}$ in dieser Phase angewandt werden. Der hydrostatische Wasserdruck unterstützt hier die RICE-Anwendung. Die Tauchbäder sind in Ruhe ohne aktive und passive Bewegung 2-mal/Tag bis auf Beckenhöhe in den ersten 2 Tage nach der Verletzung möglich [45]. Neben den o.g. Maßnahmen erwähnen einige Autoren auch noch die Manipulation des Iliosakralgelenks (ISG) mit einzubeziehen. Das ISG stellt ein wichtiges Bindeglied zwischen der Wirbelsäule und den Beinen dar. Dysfunktionen können zu Veränderung der Hamstring-Aktivität führen [27]. Insbesondere auch bei funktionellen Störungen (Typ 2A) können hier, neben der Lendenwirbelsäule, die Ursachen liegen [33]. Passive Maßnahmen wie Elektrotherapie, Iontophorese, Lymphdrainage sowie klassische Massage der gesamten Kette werden zusätzlich durchgeführt. Des Weiteren wird die krankengymnastische Übungsbehandlung mit Schwerpunkt propriozeptive neuromuskuläre Fazilitation (PNF).

Persistieren die Schmerzen über den 3.5. Tag hinaus, sollte eine erneute Evaluation der Verletzungsschwere erfolgen. Bei deutlicher Beschwerdebesserung kann der Sportler in die 2. Phase (Regenerationsphase) übergeleitet werden (Abb. 4).

\begin{tabular}{|c|c|c|}
\hline \multicolumn{3}{|c|}{ Akutphase } \\
\hline Behandlung & Zeitdauer & Zielsetzung \\
\hline „Immobilisation“ & 3-5 Tage & $\begin{array}{l}\text { 1. Eingrenzung der Narbenbildung } \\
\text { 2. korrekte Ausrichtung der Myofibrillen }\end{array}$ \\
\hline RICE & sofort/bis zu $24 \mathrm{~h}$ & $\begin{array}{l}\text { Reduzierung von Schmerz/Blutung/ } \\
\text { „Entzündung“ }\end{array}$ \\
\hline Tauchbäder/Lymphdrainage & Tag 3- 5 & $\begin{array}{l}\text { Unterstützung des Abflusses } \\
\text { in der verletzten Extremität durch } \\
\text { u.a. hydrostatischen Druck }\end{array}$ \\
\hline $\begin{array}{l}\text { Ileosakralgelenkmanipulation/ } \\
\text { Infiltration (bei Typ 2A) }\end{array}$ & Tag 3- 5 & $\begin{array}{l}\text { Wiederherstellung der lumbosakralen } \\
\text { Funktion mit ISG }\end{array}$ \\
\hline
\end{tabular}

Abb. 3 Schaubild der Akutphase.

\begin{tabular}{|l|l|l|}
\hline \multicolumn{1}{|l|}{} & \multicolumn{2}{c|}{ Regenerationsphase } \\
\hline Variable & Test & Kriterien für nächste Phase \\
\hline Schmerz nach Verletzung & auf dem Bauch/Kniegelenk 15 Grad gebeugt & keine Schmerzen \\
\hline isolierte Kraft am langen Hebel & auf dem Bauch/Kniegelenk 15 Grad gebeugt & $<10 \%$ Differenz zur Gegenseite \\
\hline neurale Defizite/Ursachen & Slump-Test & keine Schmerzen \\
\hline Hamstringflexibilität & aktiver Knieextensionstest & $<10 \%$ Differenz zur Gegenseite \\
\hline Hüftflexorenflexibilität & und <20 Grad (absolut) \\
\hline & modifizierter Thomas-Test & $>5$ Grad oder Symmetrie \\
\hline
\end{tabular}

Abb.4 Schaubild der Regenerationsphase. 


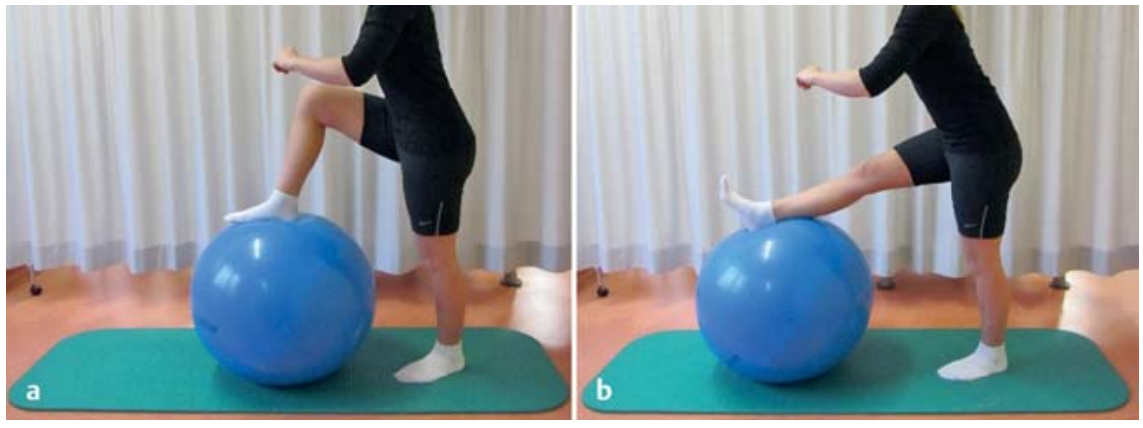

Abb. 5 Dynamische Übungen zur Verbesserung der Flexibilität der Hamstrings.
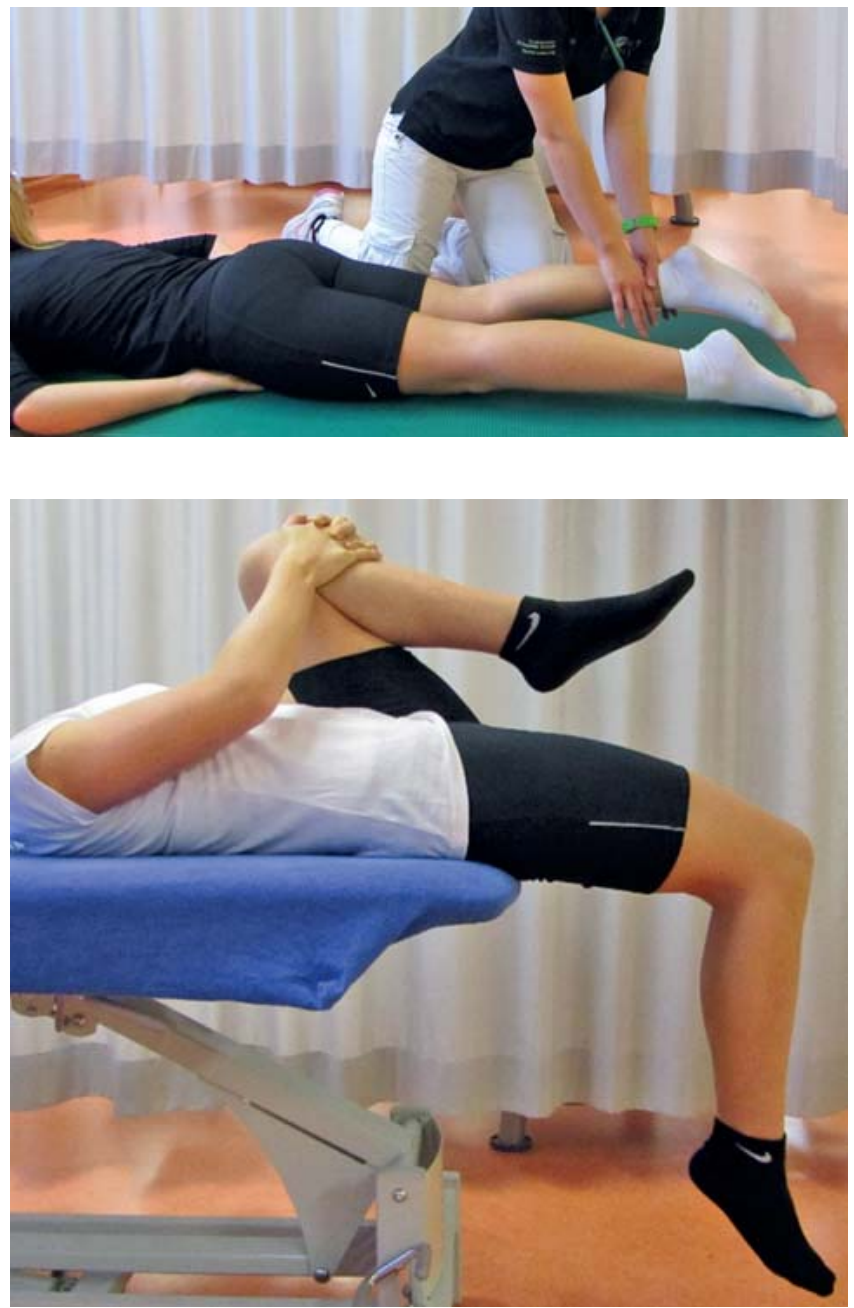

\section{Regenerationsphase}

Fibroblastenaktivität stimuliert die Produktion von Kollagen, und die einsetzende Neovaskularisierung ist die Voraussetzung für die Reparation. Leichte Querfriktion im Verletzungszentrum kann dies unterstützen. Zusätzlich stehen isometrische submaximale Übungen unterhalb der Schmerzgrenze im Mittelpunkt. Passives Dehnen unterstützt hier den Heilungsprozess [22]. Fahrradfahren und Aquajogging kann begonnen werden. Die weiteren Ziele in dieser Phase
Abb. 7 ThomasTest zur Bestimmung der Flexibilität der Hüftbeuger.

Abb. 6 Bestimmung der Kraft bei $15^{\circ}$ Flexion.

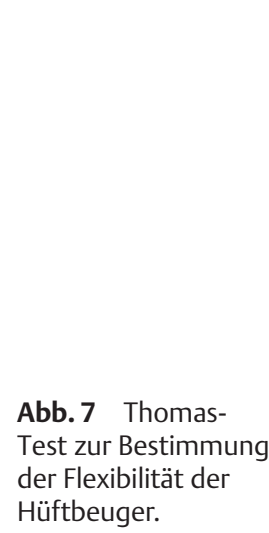

sind die Überleitung aus der Krankengymnastik in die Trainingstherapie mit allgemeiner Verbesserung der „Core Stability“, Kraftsteigerung und Schmerzreduktion während anfänglich isometrischer Übungen, Verbesserung der Flexibilität (Hamstrings/Hüftbeuger) und der neuromuskulären Kontrolle (Abb.5). Die „Core-Muskulatur“ stellt die Muskulatur um das Becken und um die untere LWS dar [3]. Diese synergistisch arbeitenden Muskeln stabilisieren Rumpf und Becken und können zu Muskelverletzungen der unteren Extremität führen
$[25,39]$. Schmerzen und Kraftdefizit sind ein häufiges Phänomen nach akuten Hamstring-Verletzungen. Die meisten Autoren empfehlen die schmerzfreie Kräftigung durch eine Steigerung von isometrischen Übungen in unterschiedlichen Flexionswinkeln mit darauffolgender konzentrischer Kräftigung. Am Ende stehen exzentrische Übungen [17]. Nur bei ausreichender Kraft bei Hamstring-Verletzungen, insbesondere für den langen Hebel, sollte der Sportler in die letzte funktionale Phase übertreten. Eine einfache Bestimmung der Kraft ist am auf dem Bauch liegenden Sportler mit $15^{\circ}$ Flexion im Kniegelenk und Seitenvergleich möglich. Ein Kraftmesser kann zusätzlich eingesetzt werden (Abb. 6) [43]. Ein weiteres Augenmerk wird auf die schnelle Wiederherstellung der Flexibilität der Hamstrings gelegt [4]. $\mathrm{Zu}$ großer Stress auf N. ischiadicus sollte dabei aber vermieden werden, da er einen möglichen Risikofaktor für die Rezidivverletzung darstellen kann [24]. Einen weiteren Risikofaktor für Rezidivverletzungen stellt die eingeschränkte Flexibilität der Hüftbeuger dar [13]. Die klinische Untersuchung kann durch den modifizierten Thomas-Test erfolgen (Abb. 7) [16]. Zur Verbesserung sollten PNF-Übungen, gefolgt von dynamischen funktionellen Dehnübungen, eingesetzt werden [28]. In Abb. 4 sind die Kriterien für die Überleitung in die funktionale Phase nochmals zusammengefasst.

\section{Funktionale Phase}

Die trainingstherapeutischen Ziele sind in der letzten Phase:

1. Vergrößern der „optimalen“ Länge der Hamstrings,

2. Verringerung der Beinasymmetrie bez. der „optimalen“ Länge der Hüftmuskulatur, der horizontalen Kraftentwicklung während des Laufens und

3. Verbesserung der Torsionsfähigkeit des Rumpfes [29].

Allgemein kann in dieser Phase mit vorsichtigem Laufbandtraining, Koordinationsschulung, Lauftraining auf dem Rasen und am Ende mit schrittweiser Wiedereingliederung in das sportartspezifische Training begonnen werden [31]. Physiotherapeutisches Ziel ist es vor allem, die Eigenschaften der sich ausbildenden Narbe auf die Anforderungen möglichst gut anzupassen (Abb. 8).

Jede Skelettmuskulatur besitzt eine optimale Länge für die Entwicklung einer 


\begin{tabular}{|l|l|l|}
\hline \multicolumn{2}{|l}{} & \multicolumn{2}{c|}{ funktionale Phase } \\
\hline Variable & Test & Kriterium für Rückkehr zum Sport \\
\hline optimaler Winkel für Spitzendrehmomente & isokinetische Knieflexion bei $60 \mathrm{Grad} / \mathrm{s}$ & $<8 \%$ gegenüber der Gegenseite \\
\hline $\begin{array}{l}\text { Hüftextensorenkraft } \\
\text { horizontale Kraft während des Laufens }\end{array}$ & $\begin{array}{l}\text { Laufen auf Laufband mit } 80 \% \\
\text { der maximalen Ausdauer }\end{array}$ & $<10 \%$ gegenüber der Gegenseite \\
\hline Kernspintomografie & Abbildung der Hamstrings & $<20 \%$ gegenüber der Gegenseite \\
\hline LWS-Rotationsstabilität & ASLR-Test & Größe des Restödems \\
\hline
\end{tabular}

Abb. 8 Schaubild der funktionalen Phase.

maximalen Kraft, die nach einer Verletzung verkürzt ist. Verletzungen entstehen wahrscheinlich, wenn der aktivierte Muskel über diese optimale Länge gedehnt wird [6]. Isokinetische Messungen zeigen valide Daten bez. Drehmoment, Geschwindigkeit, Winkelposition. Durch exzentrisches Krafttraining ist es möglich, diese optimale Länge der Muskulatur zu erreichen [7]. Für die HamstringMuskulatur bedeutet dies die Einbeziehung der Hüftbeugung bzw. Kniestreckung in der geschlossenen Kette bzw. sog. Multigelenkübungen [8]. Ein weiterer Risikofaktor für Hamstring-Verletzungen ist die nicht ausreichende Muskelkraft während exzentrischer oder konzentrischer Krafteinleitung. Insbesondere das exzentrische Spitzendrehmoment bei Knieflexion ist nach einer Hamstring-Verletzung reduziert [41]. Die reduzierte Kraft der Hüftstrecker (M. glutaeus maximus) kann ebenfalls einen Risikofaktor darstellen. Da die meisten Hamstring-Verletzungen proximal auftreten, ist die Erfassung der Hüftstrecker essenziell. Unterschiedliche einfache exzentrische Übungen [20] können zur Verbesserung der Kraft genutzt werden.

Fußballspieler können nach einer Verletzung der Hamstrings zusätzlich eine Beinasymmetrie der horizontalen Kraftentwicklung während des Laufens entwickeln. Als Gründe werden hier ein vermehrter „Becken-Tilt“ und eine veränderte Kraftübertragung von proximal nach distal in der Standphase angenommen. Die Überprüfung ist auf nicht motorisierten Laufbändern möglich. Zur Verbesserung werden uni- und bilaterale Übungen empfohlen, die dem Sportler die Möglichkeit geben, die Kraft in der horizontalen Ebene zu verbessern [9]. Die Verbesserung der Torsionsfähigkeit des Rumpfes im Hinblick auf die „Core Stability“ stellt ein weiteres wichtiges Merkmal dar. Einen sehr guten Test stellt der „Active Straight Leg Raise Test“ (ASLR) dar [26]. Gute Kontrolle ohne eine vermehrte Beckenkippung ist hier Voraussetzung.

\section{Wiedereingliederung in das sportartspezifische Training}

Die Wiedereingliederung in das sportartspezifische Training sollte individuell nach Beendigung der funktionellen Phase getroffen werden. Wichtige allgemeine Kriterien sind schmerzfreie Palpation, schmerzfreie isometrische Kontraktion, seitengleiche Beweglichkeit und nahezu gleiche Kraftentwicklung (Delta: 5-10\%) in der isokinetischen Kraftmessung zur Gegenseite [14]. Weitere spezifische Kriterien für die Hamstring-Muskulatur sind in Abb. 8 aufgeführt.

\section{Infiltrationstherapie einschließlich PRP}

Die Anwendung von autologen Produkten wie Platelet-rich Plasma (PRP) ist ein ständig und schnell wachsendes Anwendungsgebiet in der Sportmedizin. Seit 2011 stellt die Injektion von autologem PRP kein Doping mehr dar [47]. Gegenüber dem normalen Serum ist die Konzentration der Thrombozyten im PRP deutlich höher (mindestens: 1000000 platelets/ $\mu \mathrm{l}$ in $5 \mathrm{ml}$ ) und die Konzentration von Wachstumsfaktoren steigt auf das 3- bis 5-Fache. Es wird angenommen, dass die Wachstumsfaktoren die Muskelregeneration beeinflussen und die Rehabilitationszeit verkürzen können. Obwohl viele Grundlagen- und Tierstudien hierzu veröffentlicht sind, gibt es noch keine randomisierten kontrollierten Studien, die die Überlegenheit von PRP bei Muskelverletzungen gegenüber einer Kontrollgruppe bestätigen. Meist handelt es sich um retrospektive oder Fall-Kontroll-Studien ohne Randomisierung und Kontrollgruppe [1]. Hammond et al. zeigten im Tiermodell, dass der Ein- satz von PRP signifikant die Heilungszeit von 21 auf 14 Tage reduzierte [15]. Sanchez et al. berichteten 2005 auf dem 2. „World Congress on Regenerative Medicine 2005“ über ähnliche Ergebnisse. Sie zeigten, dass sich die Rehabilitationszeit halbierte [38]. In einer Arbeit von Wright-Carpenter et al. wurde die „Return-to-Sport-Zeit“ um 30\% (6 Tage) vermindert. In einer Arbeit aus dem Jahre 2013 zeigten Wetzel et al. in einer retrospektiven Arbeit über 17 Sportler, dass es auf der visuellen Analogskala zu einer signifikanten Verbesserung kam [44]. Das Internationale Olympische Komitee (IOC) kommt 2010 zu dem Schluss, dass es im Moment nur wenig wissenschaftlich belegte Ergebnisse für den Einsatz von PRP bei Sportverletzungen gibt [12]. In einem systematischen Review aus dem Jahre 2011 zeigen Andia et al. ebenfalls, dass es keine randomisierten Studien gibt, die den Vorteil von PRP-Injektionen bei Muskelverletzungen bestätigen [1]. Das einzige uns bekannte randomisierte Studienprotokoll wurde von Hamid et al. 2012 veröffentlicht. Partialrupturen der Hamstrings wurden hier eingeschlossen und in 2 Gruppen randomisiert. Beide Gruppen erhielten ein standardisiertes Physioprogramm, eine Gruppe erhält zusätzlich die Injektion von PRP.

Unter Berücksichtigung der genannten Ergebnisse kann die Therapie mit PRP in bestimmten Fällen und in Kombination mit physikalischen Therapiemaßnahmen eine Option darstellen. Wir verwenden PRP im Moment bei Grad-IIIbVerletzungen. Der Einsatz von PRP sollte aber nicht für eine Beschleunigung der einzelnen Heilungsphasen, sondern für die optimale Heilung im zeitlichen Rahmen gesehen werden $[21,33]$.

Die Injektion erfolgt unter sterilen Bedingungen und Ultraschallkontrolle. Ein geringes Volumen von Lokalanästheti- 


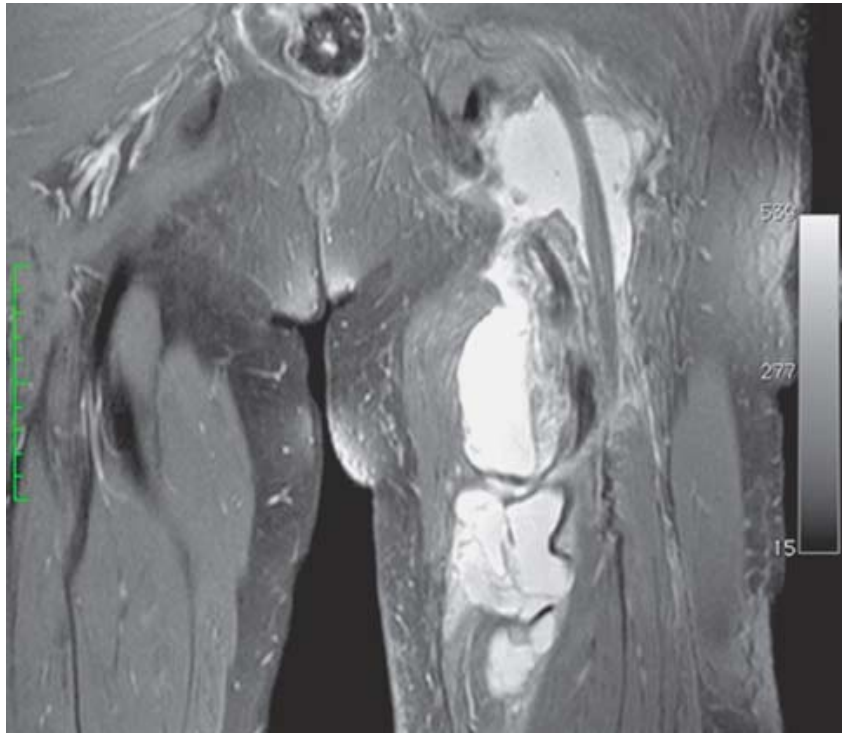

Abb. 9 Koronare FS-Aufnahme mit komplettem Abriss der HamstringMuskulatur. Teile des Bizepsmuskels stehen noch.

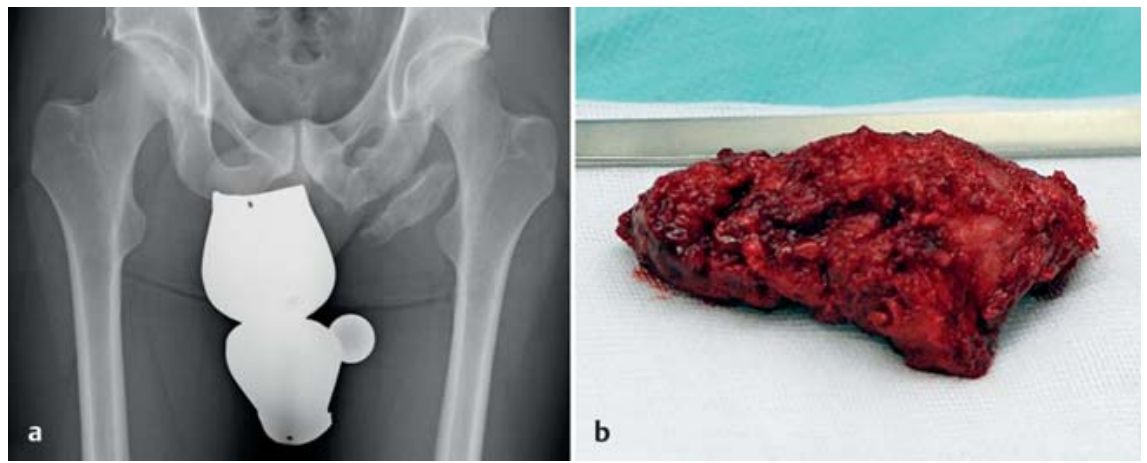

Abb.10a und b Ausbildung einer ausgeprägten Ossifikation nach Hamstring-Verletzung: a Röntgenbild, b Operatives Korrelat.

kum wird proximal und distal der Ruptur injiziert. Ein evtl. vorhandenes Hämatom wird aspiriert und das PRP-Serum ( $3 \mathrm{ml}$ ) wird injiziert. Eine elastokompressive Wickelung steht am Schluss der Behandlung und der Sportler wird aufgefordert, für 20-30 Minuten in sitzender oder halb sitzender Haltung zu ruhen.

Die Infiltrationstherapie nach MüllerWohlfahrt [31] ist in der sportmedizinischen Praxis in Deutschland weit verbreitet [48]. Insbesondere bei funktionellen Störungen vom Typ 1 und 2 (Tab.1) können sie adjuvant eingesetzt werden. Das Standardregime besteht, je nach Ausmaß der Muskelverletzung, aus Injektion von Lokalanästhekium, Actovegin und Traumeel S an den Tagen 0, 2 und 4 nach der Verletzung. Injektionen erfolgen an die verletzte Stelle selbst, in einer vertikalen Linie entlang des verletzten Muskels und im Bereich der Lendenwirbelsäule bei neurogen bedingten Störungen (Typ 2A). Wie bei dem Einsatz von PRP fehlt auch hier der wissenschaftliche Beweis. Es bestehen aber Pa- rallelen zwischen dem Verständnis der Muskelheilung durch Müller-Wohlfahrt und der Grundlagenforschung, die einen Nutzen in einer gewissen Kontrolle der Entzündungsphase sehen [5]. Weltweit anerkannte Sportmediziner kommen deshalb auf einem sportmedizinischen Expertenmeeting 2007 in London zu dem Schluss, dass die genannte Infiltrationstherapie eine gute Therapieoption darstellen kann.

\section{Operative Therapie}

Die operative Versorgung von Muskelverletzungen ist seltenen Indikationen vorbehalten:

- großes Hämatom

- komplette/subtotale Ruptur (Abb.9)

- Verletzungen mit mehr als 50\% des Durchmessers/der Fläche

- schmerzhafte ausgeprägte Narbenbildung mit deutlichem Extensionsdefizit

- schmerzhafte Myositis ossificans

- evtl. großzügiger, falls keine Synergisten $[23,24]$
Die Operation sollte möglichst schnell nach der akuten Verletzung erfolgen. Der Vorteil ist zusätzlich in der Frühmobilisation zu sehen. Hämatom und nekrotisches Gewebe sollten sorgfältig entfernt werden. Alternativ kann das Hämatom, wenn möglich, durch Punktion entlastet werden [36]. Die Muskelnähte erfolgen mit resorbierbarem Material optimalerweise in einer modifizierten Technik nach Kragh [23]. Die verletzte Stelle wird hier in 4 Quadranten eingeteilt. Unter Fassen des Epimysiums erfolgen vorsichtige umgreifende Nähte. Danach erfolgt die adaptierende Naht in modifizierter Mason-Allen-Naht. Die umgreifenden Nähte sollten hier nicht $\mathrm{zu}$ fest gezogen werden, um das darunter liegende Muskelgewebe nicht noch zusätzlich zu schädigen. Sind mehrere Muskelfaszien betroffen, sollten sie einzeln rekonstruiert werden. Die operative Therapie am muskulotendinösen Übergang ergibt bessere Ergebnisse.

Wie oben schon erwähnt, sind die Hamstrings die häufigste verletzte Muskelgruppe. Mehrere Studien zeigten, dass durch frühzeitige Operation von kompletten proximalen Abrissverletzungen gute funktionelle Ergebnisse und eine hohe Rückkehrrate zum Sport erreicht werden kann [46]. Ebenso verhält es sich bei Verletzungen der proximalen Anteile des M. rectus femoris [40]. Fadenankerrefixationen stellen hier eine gute Operationsmethode dar. Die postoperative Lagerung erfolgt in „Neutralposition“ mithilfe einer Orthese. Die Zeitdauer der Immobilisation hängt von der Größe der Verletzung und dem Rekonstruktionsergebnis ab. In der Regel erfolgt eine Teilbelastung für 4-6 Wochen mit vorsichtigen passiven Dehnübungen. Hier ist anfänglich auf eine spannungsfreie Hüftflexion von $30-60^{\circ}$ bei gleichzeitiger Knieflexion zu achten. Mit dem Beginn der 4. Woche kann die Hüftflexion auf $60-90^{\circ}$ gesteigert werden. Die Vollbelastung und Mobilisation kann ab der 7. Woche erreicht werden.

Schmerzhafte Ossifikationen als Folge einer Metaplasie bei großem Hämatom können im chronischen Fall eine Operation notwendig machen (Abb.10). Bei chronischen Verletzungen stehen meist die Mobilisation der Narbe und die Wiederherstellung der Muskelkontinuität im Vordergrund. Die Ergebnisse sind im Vergleich zur Akutversorgung aber schlechter [37]. 


\section{Literatur}

${ }^{1}$ Andia I, Sanchez M, Maffulli N. Platelet rich plasma therapies for sports muscle injuries: any evidence behind clinical practice? Expert Opin Biol Ther 2011; 11: 509-518

2 Bleakley C, McDonough S, MacAuley D. The use of ice in the treatment of acute soft-tissue injury a systematic review of randomized controlled trials. Am J Sports Med 2004; 32 : 251-261

${ }^{3}$ Bliss LS, Teeple P. Core stability: the centerpiece of any training program. Curr Sports Med Rep 2005; 4: 179-183

${ }^{4}$ Bradley PS, Portas MD. The relationship between preseason range of motion and muscle strain injury in elite soccer players. J Strength Cond Res 2007; 21: 1155

${ }^{5}$ Brickson S, Ji LL, Schell K et al. M1/70 attenuates blood-borne neutrophil oxidants, activation, and myofiber damage following stretch injury. J App Physiol 2003; 95: 969-976

${ }^{6}$ Brooks JH, Fuller CW, Kemp SP et al. Incidence, risk, and prevention of hamstring muscle injuries in professional rugby union. Am J Sports Med 2006; 34: 1297-1306

${ }^{7}$ Brughelli M, Cronin J. Altering the length-tension relationship with eccentric exercise: implications for performance and injury. Sports Med 2007; 37: 807-826

8 Brughelli M, Cronin J. Preventing hamstring injuries in sport. Strength Cond J 2008; 30: 55

9 Brughelli M, Cronin J, Mendiguchia J et al. Contralateral leg deficits in kinetic and kinematic variables during running in Australian rules football players with previous hamstring injuries. J Strength Cond Res 2010; 24: 25392544

10 Ekstrand J, Healy JC, Walden M et al. Hamstring muscle injuries in professional football: the correlation of MRI findings with return to play. Br J Sports Med 2012; 46: 112 117

${ }^{11}$ Elliott MC, Zarins B, Powell JW et al. Hamstring muscle strains in professional football players: a 10-year review. Am J Sports Med 2011; 39: 843-850

12 Engebretsen L, Steffen K, Alsousou J et al. IOC consensus paper on the use of platelet-rich plasma in sports medicine. Br J Sports Med 2010; 44: 1072-1081

13 Gabbe BJ, Bennell KL, Finch CF. Why are older Australian football players at greater risk of hamstring injury? J Sci Med Sport 2006; 9: 327-333

14 Hamilton B, Knez W, Eirale Cet al. Platelet enriched plasma for acute muscle injury. Acta Orthop Belg 2010; 76: 443-448

15 Hammond JW, Hinton RY, Curl LA et al. Use of autologous platelet-rich plasma to treat muscle strain injuries. Am J Sports Med 2009; 37: 1135-1142

${ }^{16}$ Harvey D. Assessment of the flexibility of elite athletes using the modified Thomas test. Br J Sports Med 1998; 32: 68-70

${ }^{17}$ Heiderscheit BC, Sherry MA, Silder $A$ et al. Hamstring strain injuries: recommendations for diagnosis, rehabilitation and injury prevention. J Orthop Sports Phys Ther 2010; 40: 67-81

18 Jarvinen MJ, Lehto MU. The effects of early mobilisation and immobilisation on the healing process following muscle injuries. Sports Med 1993; 15: 78-89

19 Jarvinen TA, Jarvinen TL, Kaariainen $M$ et al. Muscle injuries: biology and treatment. Am J Sports Med 2005; 33: 745-764

20 Jonhagen S, Ackermann P, Saartok T. Forward lunge: a training study of eccentric exercises of the lower limbs. I Strength Cond Res 2009; 23: 972-978

${ }^{21}$ Kasemkijwattana C, Menetrey J, Bosch P et al. Use of growth factors to improve muscle healing after strain injury. Clin Orthop Relat Res 2000; 370: 272-285

${ }^{22}$ Khan K, Scott A. Mechanotherapy: how physical therapists' prescription of exercise promotes tissue repair. Br J Sports Med 2009; 43: 247-252

${ }^{23}$ Kragh JF jr., Basamania CJ. Surgical repair of acute traumatic closed transection of the biceps brachii. J Bone Joint Surg Am 2002; 84: 992-998

${ }^{24}$ Kujala U, Orava S, Järvinen M. Hamstring injuries. Current trends in treatment and prevention. Sports Med 1997; 23: 397

${ }^{25}$ Leetun DT, Ireland ML, Willson JD et al. Core stability measures as risk factors for lower extremity injury in athletes. Med Sci Sports Exerc 2004; 36: 926-934

${ }^{26}$ Liebenson C, Karpowicz AM, Brown SH et al. The active straight leg raise test and lumbar spine stability. PM R 2009; 1: 530-535

27 Mason D, Dickens V, Vail A. Rehabilitation for hamstring injuries. Scand J Med Sci Sports 2007; 17: 191-192

${ }^{28}$ McGill S. Low Back Disorders: Evidence-based Prevention and Rehabilitation. Champaign, IL: Human Kinetics Publishers; 2007

${ }^{29}$ Mendiguchia J, Brughelli M. A return-to-sport algorithm for acute hamstring injuries. Phys Ther Sport 2011; 12: 2-14

${ }^{30}$ Mueller-Wohlfahrt HW, Haensel L, Mithoefer K et al. Terminology and classification of muscle injuries in sport: the Munich consensus statement. Br J Sports Med 2013; 47: 342350

31 Müller-Wohlfahrt HW, Hänsel L, Ueblacker P. Muskelverletzungen im Sport. Stuttgart: Thieme; 2010

32 Orchard J, Read J, Neophyton J et al. Groin pain associated with ultrasound finding of inguinal canal posterior wall deficiency in Australian Rules footballers. Br J Sports Med 1998; 32: 134-139

33 Orchard JW, Best TM, Mueller-Wohlfahrt HW et al. The early management of muscle strains in the elite athlete: best practice in a world with a limited evidence basis. Br J Sports Med 2008; 42: 158-159

34 Paoloni JA, Orchard JW. The use of therapeutic medications for soft-tissue injuries in sports medicine. Med J Aust 2005; 183: 384-388

35 Peetrons $P$. Ultrasound of muscles. Eur Radiol 2002; $12: 35-43$
${ }^{36}$ Peterson L, Renström P, Rost G et al. Verletzungen im Sport: Handbuch der Sportverletzungen und Sportschäden für Sportler, Übungsleiter und Ärzte. Köln: Deutscher Ärzte-Verlag; 1987

37 Sallay PI, Friedman RL, Coogan PG et al. Hamstring muscle injuries among water skiers. Functional outcome and prevention. Am J Sports Med 1996; 24: 130-136

38 Sanchez M, Anitua E, Andia I. Application of autologous growth factors on skeletal muscle healing. In: 2nd World Congress on Regenerative Medicine. Podium Presentation; 2005

${ }^{39}$ Sherry MA, Best TM. A comparison of 2 rehabilitation programs in the treatment of acute hamstring strains. J Orthop Sports Phys Ther 2004; 34: 116-125

${ }^{40}$ Straw R, Colclough K, Geutjens G. Surgical repair of a chronic rupture of the rectus femoris muscle at the proximal musculotendinous junction in a soccer player. Br J Sports Med 2003; 37: 182-184

${ }^{41}$ Sugiura Y, Saito T, Sakuraba K et al. Strength deficits identified with concentric action of the hip extensors and eccentric action of the hamstrings predispose to hamstring injury in elite sprinters. J Orthop Sports Phys Ther 2008; 38: 457-464

42 Volpi P, Melegati G, Tornese D et al. Muscle strains in soccer: a five-year survey of an Italian major league team. Knee Surg Sports Traumatol Arthrosc 2004; 12: 482-485

43 Warren P, Gabbe BJ, Schneider-Kolsky M et al. Clinical predictors of time to return to competition and of recurrence following hamstring strain in elite Australian footballers. $\mathrm{Br}$ J Sports Med 2010; 44: 415-419

44 Wetzel R, Patel R, Terry M. Platelet-rich plasma as an effective treatment for proximal Hamstring injuries. Orthopedics 2013; 36: e64

45 Wilcock IM, Cronin JB, Hing WA. Physiological response to water immersion: a method for sport recovery? Sports Med 2006; 36: 747 765

${ }^{46}$ Wood DG, Packham I, Trikha SP et al. Avulsion of the proximal hamstring origin. J Bone Joint Surg Am 2008; 90: 2365-2374

47 World Anti Doping Agency. List of prohibited substances and methods. 2013. Im Internet: http://list.wada-ama.org; Stand: Mai 2016

48 Wright-Carpenter T, Klein P, Schäferhoff P et al. Treatment of muscle injuries by local administration of autologous conditioned serum: a pilot study on sportsmen with muscle strains. Int J Sports Med 2004; 25: 588-593

\section{Dr. med. Frieder Mauch M. A.}

Chefarzt

Sportklinik Stuttgart

Taubenheimstraße 8

70372 Stuttgart

frieder.mauch@sportklinik-stuttgart.de 